\title{
Structural and magnetic investigations on $\mathrm{Ni}_{2} \mathrm{MnAl}$
}

\author{
LI. Mañosa, A. Planes, Ch. Somsen ${ }^{1}$, Ch. Fell ${ }^{1}$ and M. Acet ${ }^{1}$ \\ Departament d'Estructura i Constituents de la Matèria, Facultat de Física, \\ Universitat de Barcelona, Diagonal 647, 08028 Barcelona, Catalonia, Spain \\ ${ }^{1}$ Tieftemperaturphysik, Gerhard-Mercator Universität Duisburg, 47048 Duisburg, Germany
}

\begin{abstract}
Ferromagnetic alloys undergoing thermoelastic martensitic transformations have recently become very attractive owing to the possibility of inducing giant deformations by rotating martensitic domains with an externally applied magnetic field. It is expected that ferromagnetic alloys with the Heusler structure may exhibit a strong magnetoelastic coupling resulting in potentially interesting magneto-mechanical properties. In this paper we present preliminary results on structural and magnetic studies on $\mathrm{Ni}_{2} \mathrm{MnAl}$. It is shown that in this material the magnetic properties strongly depend on the heat treatment, chiefly because of the difficulty in achieving a complete $\mathrm{L} 2$, order.
\end{abstract}

\section{INTRODUCTION}

The shape-memory effect occurring in a number of alloys is associated with the thermoelastic martensitic transformation and has proven to be of significant technological interest. Over the years, many devices have been developed which rely both on the one-way and the two-way shape memory effects. However, one of the major inconveniences that all these devices have is the slow response, which is inherent to the thermal control of the martensitic transformation. A possible way for circumventing such an inconvenience has recently been envisaged. The existence of ferromagnetic shape-memory alloys, for which a shape change is achieved by the application of a magnetic field, seems to open up the possibility of developing magnetic actuators [1].

Until now, the only system which has been shown to exhibit such an interesting property is Ni-Mn-Ga with compositions close to that of the Heusler compound $\mathrm{Ni}_{2} \mathrm{MnGa}$. These alloys possess an $\mathrm{L} 2{ }_{1}$ structure that transforms martensitically on cooling. The magnetic coupling in both the parent and the product phase is ferromagnetic. In the martensitic phase, the combination of a relatively large magnetic anisotropy and a strong magnetoelastic coupling enables the rotation of martensite domains under the application of a magnetic field [2]. From the point of view of practical application however, this alloy has the drawback of being brittle.

The search for materials other than Ni-Mn-Ga which are potentially able to exhibit magnetic shape memory effect seems to be of capital importance. Good candidates are Ni-Mn-Al alloys. Already a number of investigations aimed at determining the properties of this alloy system [3-6] have been undertaken. However, the results reported differ considerably from one another. This appears to originate from the extreme sensitivity of the properties of this material to heat treatment, which varies amongst different groups.

Earlier X-ray diffraction studies have shown that above $1220 \mathrm{~K}$ the alloy $\mathrm{Ni}_{2} \mathrm{MnAl}$ is in a disordered bec phase (A2). Below this temperature the system undergoes a second order transformation into the B2 phase. Below about $1000 \mathrm{~K}$ there is another onset of a second order transformation into the L2, Heusler phase [7]. Before one can understand the properties of the martensitic transformation, for which the properties are very sensitive to composition, a thorough understanding of the physical properties of the stoichiometric composition is required. In this work we undertake a study of the structural and magnetic behavior of $\mathrm{Ni}-\mathrm{Mn}-\mathrm{Al}$ alloys and consider the as-prepared state and the state after quenching from $1373 \mathrm{~K}$. We report on preliminary results on the magnetic behavior of the stoichiometric compound $\mathrm{Ni}_{2} \mathrm{MnAl}$.

\section{EXPERIMENT}

The $\mathrm{Ni}_{2} \mathrm{AlMn}$ sample was prepared by induction melting. EDX analysis gave an actual concentration of $\mathrm{Ni}_{50.4} \mathrm{Mn}_{24.7} \mathrm{Al}_{24.9}$. Samples of appropriate sizes were cut from the resulting ingot for AC susceptibility $\chi_{\mathrm{ac}}$ and 
DC susceptibility $\chi_{\mathrm{dc}}$ measurements. For transmission electron microscopy (TEM), $3 \mathrm{~mm}$ diameter and $250 \mu \mathrm{m}$ thick samples with were prepared by electrochemical etching using an acid solution [8]. The experiments were carried out on samples that were simply left to cool to room temperature after melting (as-prepared) and on samples that were quenched to $0^{\circ} \mathrm{C}$ after having been annealed at $1100^{\circ} \mathrm{C}(1373 \mathrm{~K})$ for three days within the $\mathrm{A} 2$ phase. In the temperature range $300 \mathrm{~K} \leq \mathrm{T} \leq 900 \mathrm{~K}$ the $\chi_{\mathrm{dc}}$ was measured with a vibrating sample magnetometer. The measurements below room temperature were carried out using a SQUID magnetometer. $\chi_{\mathrm{ac}}$ was measured in the temperature range $80 \mathrm{~K} \leq \mathrm{T} \leq 300 \mathrm{~K}$ in a field of 25 Oe with a frequency of $666 \mathrm{~Hz}$. The specific heat measurements were carried out using a modulated differential scanning calorimeter in the temperature range $200 \mathrm{~K} \leq \mathrm{T} \leq 350 \mathrm{~K}$.

\section{RESULTS}

\subsection{Electron microscopy}

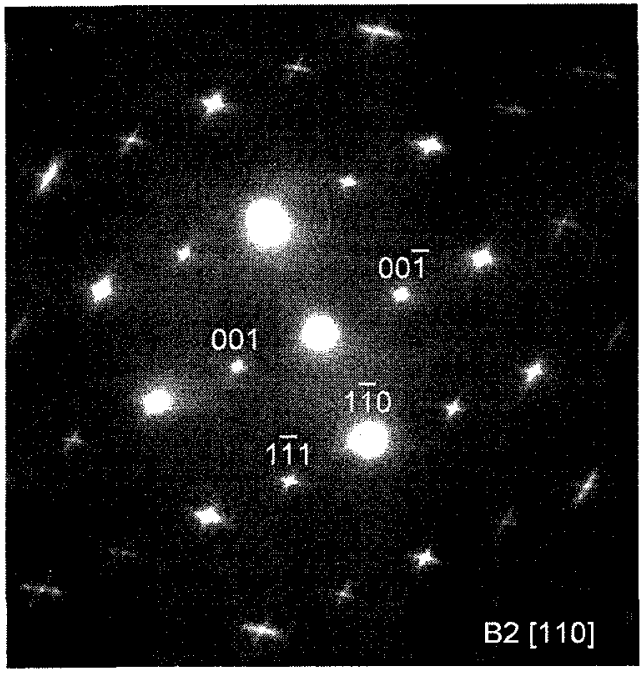

(a)

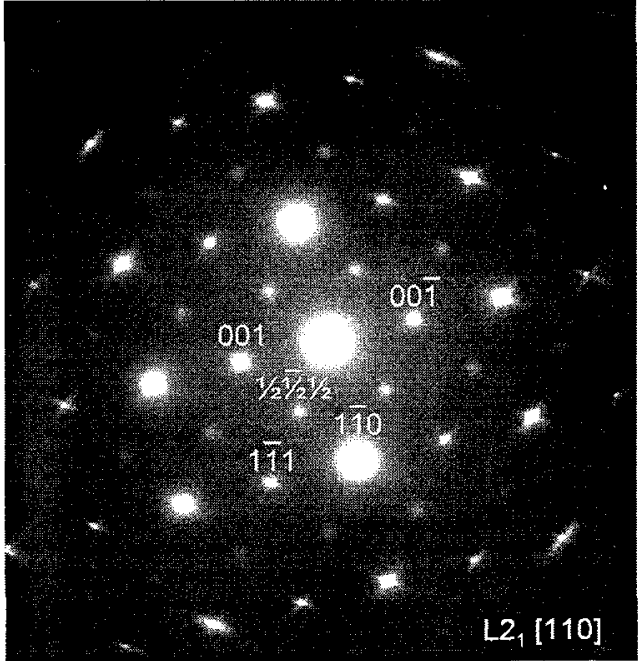

(b)

Figure 1. Electron diffraction patterns of (a) the $\mathrm{B} 2$ and (b) the $\mathrm{L}_{1}$ phase of $\mathrm{Ni}_{2} \mathrm{MnAl}$ of the [110] zone. The indices in $\mathrm{B} 2$-phase notation refer to the spots underneath.

The results of the TEM observations are given with the electron diffraction images in figure 1a and figure $1 \mathrm{~b}$ for the sample in the state quenched from $1373 \mathrm{~K}$ within the A2 stability range and in the as-prepared state respectively. Both patterns relate to the [110] zone. The image in figure 1a is characteristic of the B2 phase indicating that the $\mathrm{A} 2$ phase is not retained with the quenching rate used in this experiment. However, from this figure alone one cannot exclude the possibility of some amount of coexisting A2 phase. The presence of an $L 2$ phase is not observable in this image. The sample in the as-prepared state gives the image in figure $2 b$ which is characteristic of the $\mathrm{L} 2{ }_{1}$ phase observed in Heusler alloys. However, the possibility of a coexisting B2 phase cannot be excluded. The streaking of the spots in both figures are eventually observed as crosses when moved to the outer parts of the images. Such streaking has also been observed in Ni-Mn-Al samples with slightly different compositions [3], and also in other Ni-based shape-memory alloys [7]. This has been ascribed to the existence of modulated structures (tweed-pattern) associated with a premartensitic state in the cubic phase at temperatures well above the nominal martensitic transition temperature. Although the stoichiometric alloy does not undergo a martensitic transformation, its composition lies close to the transformation boundary so that the origin of such streaking can be due to similar causes. 

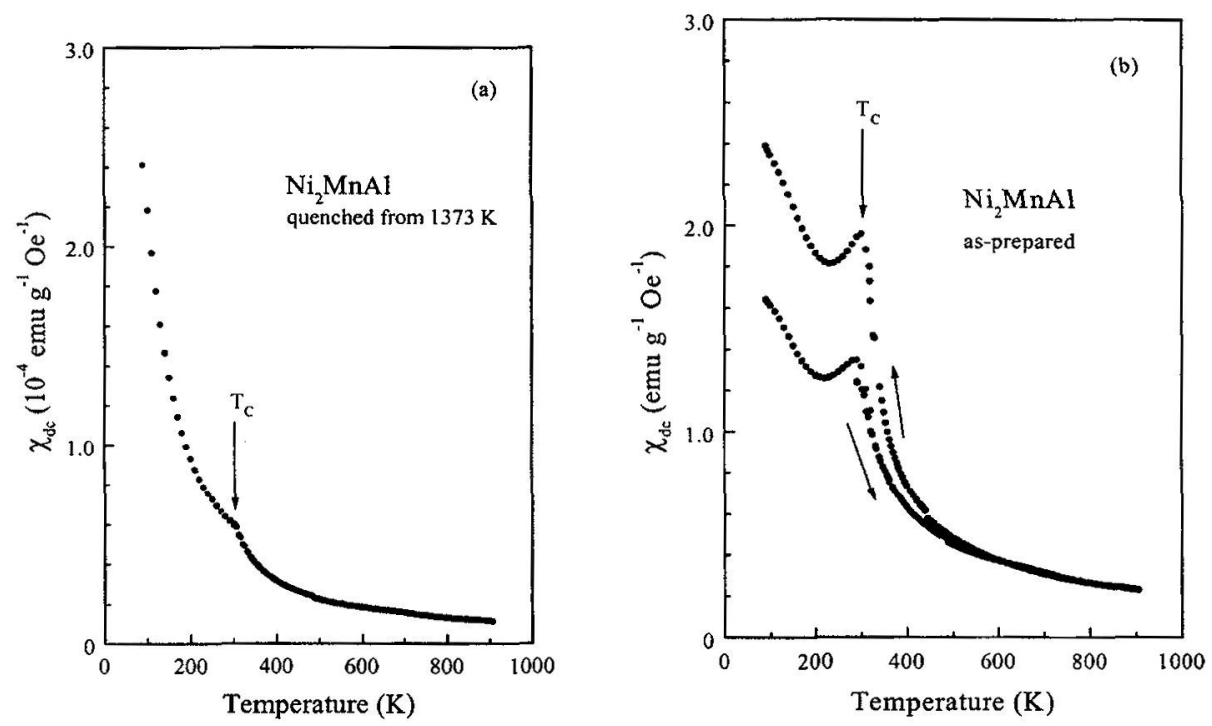

Figure 2. $\chi_{\mathrm{dc}}(\mathrm{T})$ of $\mathrm{Ni}_{2} \mathrm{MnAl}$ in the (a) quenched and (b) as prepared states. The arrows in part (b) indicate the temperature change direction.

\subsection{DC susceptibility and the magnetization}

The temperature dependence of $\chi_{\mathrm{dc}}$ for the quenched and annealed samples are shown in figure $2 \mathrm{a}$ and $2 \mathrm{~b}$ respectively. $\chi_{\mathrm{dc}}$ of the quenched sample, which is essentially in the $\mathrm{B} 2$ phase, decreases with increasing temperature and at a critical magnetic transition temperature $T_{C} \simeq 300 \mathrm{~K}$ the curve exhibits an abrupt change of slope before resuming to decrease progressively. The features in the temperature dependence of $\chi_{\mathrm{dc}}$ of the as prepared sample shown in figure $2 \mathrm{~b}$ are somewhat different. On increasing temperature $\chi_{\mathrm{dc}}$ initially decreases and then passes through a maximum at $T_{C}$ where the magnetic transition is seen to be more pronounced than in the quenched state. $\chi_{\mathrm{dc}}$ then resumes decreasing on further increasing the temperature.

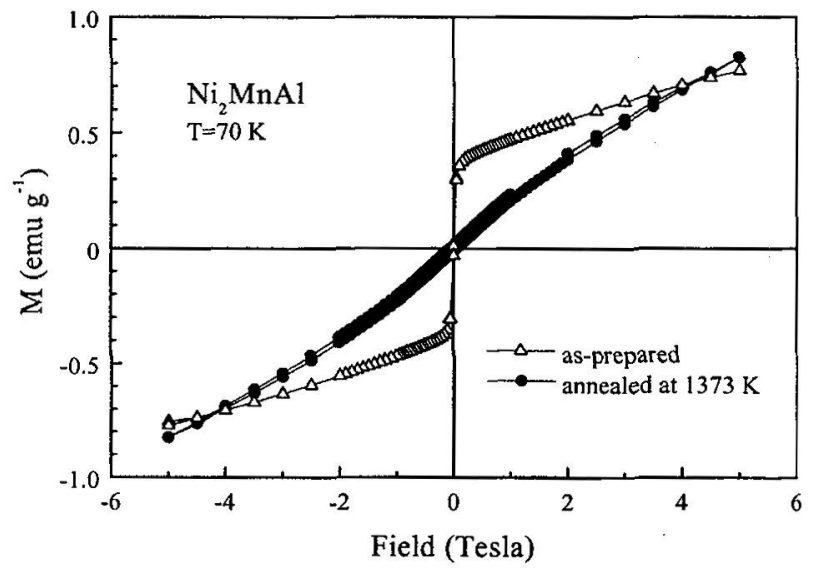

Figure 3. The field dependence of the magnetization of the quenched and as-prepared samples at $70 \mathrm{~K}$. 

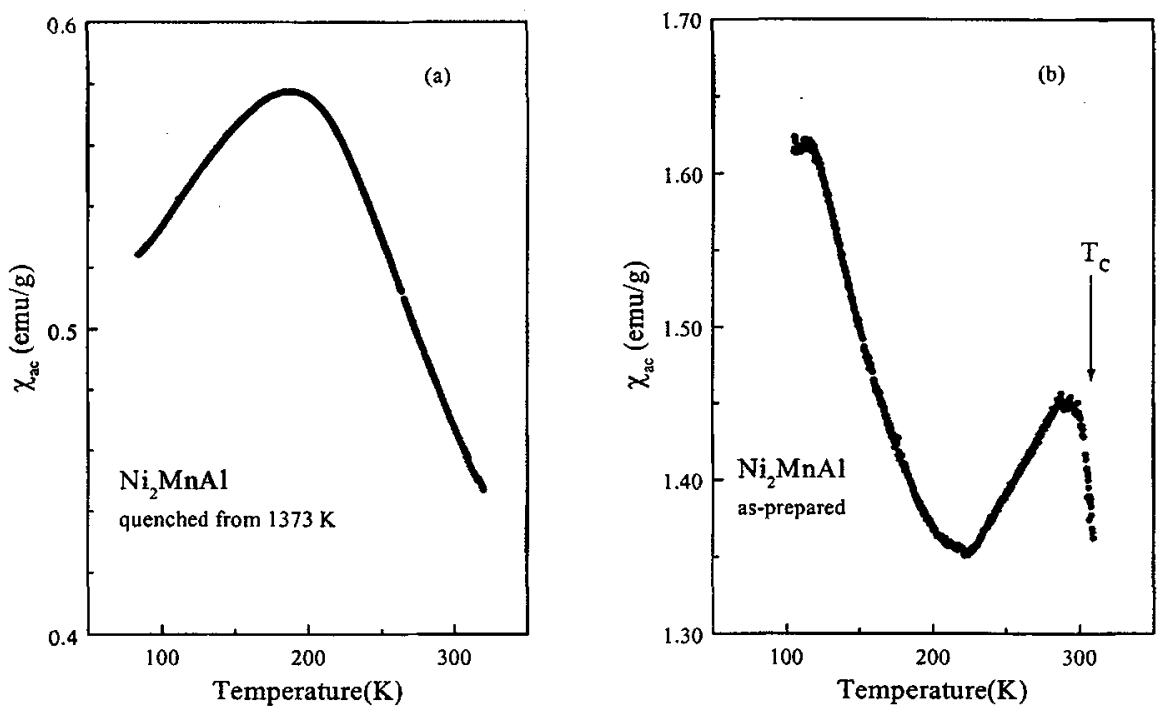

Figure 4. The temperature dependence of the real part of the ac susceptibility for the (a) annealed and (b) as-prepared sample.

In the process of heating above $600 \mathrm{~K}$ diffusion effects become effective and any frozen-in $\mathrm{B} 2$ entity in the as-prepared sample begins to decompose leaving its presence to the more stable $L 2_{1}$ phase. On recooling from $900 \mathrm{~K}$ the decomposition progresses back down to $600 \mathrm{~K}$. This portion of the measurement lasts about 12 hours. The new state, which is still a B2-L2 ${ }_{1}$ mixture but with more L2 ${ }_{1}$ components than the as-prepared state exhibits a larger susceptibility with the essential features remaining similar to the heating curve.

The field dependence of the magnetization up to $6 \mathrm{~T}$ is shown in figure 3 . Neither the magnetization of the as-prepared nor the annealed sample approaches saturation at high fields. The magnetization of the annealed sample deviates from linearity, but changes smoothly in the measured field range. The as-prepared sample exhibits a fast reversal of the magnetization at small fields indicating to the presence of ferromagnetic

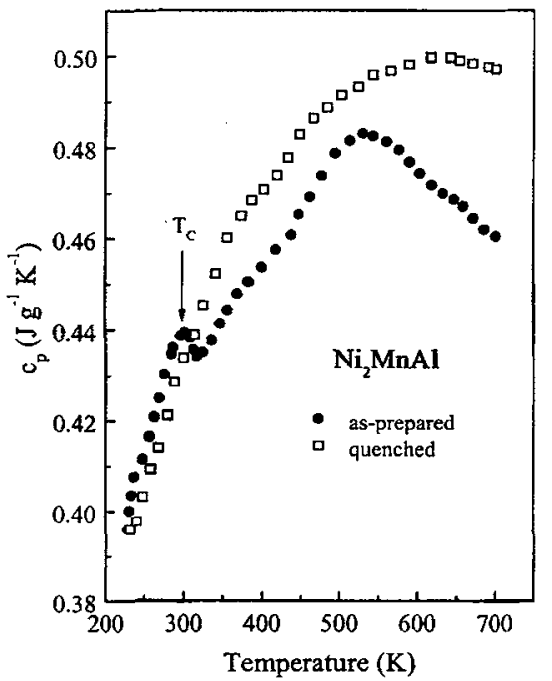

Figure 5. The temperature dependence of the specific heat of $\mathrm{Ni}_{2} \mathrm{MnAl}$ in the two sample preparation states. components.

\subsection{AC susceptibility}

Figures $4 \mathrm{a}$ and $4 \mathrm{~b}$ show the temperature dependence of the real part of $\chi_{\mathrm{ac}}$ for the samples under the two preparation conditions. The features in these two figures are also quite different. In the quenched state of the sample $\chi_{\mathrm{ac}}$ first increases with increasing temperature and goes through a maximum, after which it decreases continuously. There is no indication of a magnetic transition around $300 \mathrm{~K}$. On the other hand $\chi_{\mathrm{ac}}$ of the as-prepared sample first decreases with increasing temperature and goes through a minimum around $220 \mathrm{~K} . \chi_{\mathrm{ac}}$ then increases and subsequently drops rapidly as $\mathrm{T}_{\mathrm{C}}$ is approached.

\subsection{Specific heat}

The temperature dependence of the specific heat $c_{p}$ for both sample states is shown in figure $5 . c_{p}$ of the quenched sample increases steadily with increasing temperature up to about $600 \mathrm{~K}$ above which diffusion effects become 


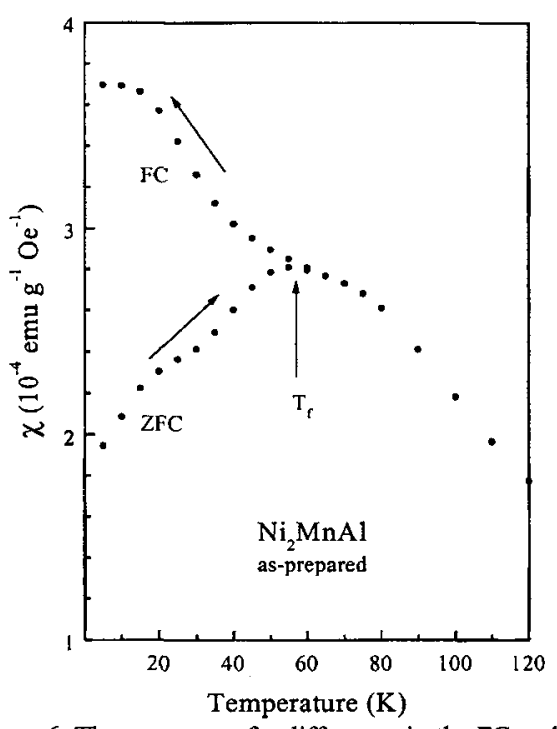

Figure 6. The presence of a difference in the $\mathrm{FC}$ and ZFC susceptibilities showing the spin-glass nature of the as-prepared $\mathrm{Ni}_{2} \mathrm{MnAl}$ sample at low temperatures. effective, by which the $\mathrm{L} 2{ }_{1}$ phase begins to stabilize and the measurements are no longer in equilibrium, as also observed in the $\chi_{\mathrm{dc}}$ data in figure $2 . c_{\mathrm{p}}$ of the as-prepared sample on the other hand shows a feature around $300 \mathrm{~K}$ which corresponds to $T_{C}$ observed in the magnetic measurements.

\section{DISCUSSION}

The measured parameters in the present work show that the features pertaining to the magnetic transition observed in the data of the $\mathrm{L} 2$, phase strongly fade, and they are practically nonexistent in the B2 phase. Although the B2 phase appears not to be responsible for the magnetic ordering effects, the value of $\chi_{\mathrm{dc}}$ is still comparable to that of the L2, phase.

The occurrence of magnetic ordering in the $L 2$ phase is in good agreement with earlier investigations $[5,6,10]$. Earlier neutron diffraction experiments at $4 \mathrm{~K}[10]$ had given evidence for antiferromagnetic ordering. Antiferromagnetic components no doubt exist due to the occurrence of a

spin-glass-like frustrated state at low temperatures, as seen by the difference of the magnetic susceptibilities in the field-cooled (FC) and zero-field-cooled (ZFC) states in figure 6. On the other hand, the occurrence of ferromagnetic components, required for the presence of the spin glass state, is evidenced in the magnetization curve in figure 3. Presently, it is not possible to give a clear description of the nature of magnetic ordering occurring just below $\mathrm{T}_{\mathrm{C}}$. A sample prepared wholly in the $\mathrm{L} 22_{1}$ phase is required for such a study.

\section{Acknowledgments}

This work was partially supported by DGESIC (Spain) and DAAD (Germany).

\section{References}

1. K. Ullakko, J.K. Huang, C. Kantner, R.C. O'Handley, V. V. Kokorin, Appl. Phys. Lett. 69, 1966 (1996).

2. Ll. Mañosa, A. Planes, Adv. in Solid State Phys. 2000 (in press), and references therein.

3. R. Kainuma, H. Nakano, K. Ishida, Metall. and Mater. Trans. 27A, 4513 (1996).

4. Y. Sutou, I. Ohnuma, R. Kainuma, K. Ishida, Metall. and Mater. Trans. 29A, 222 (1998).

5. F. Gejima, Y. Sutou, R. Kainuma, K. Ishida, Metall. and Mater. Trans. 30A, 2721 (1999).

6. S. Morito, T. Kakeshita, K. Hirata, K. Otsuka, Acta Met. 46, 5377 (1998).

7. J. Soltys, phys. stat. sol. (a) 66,485 (1981).

8. D. B. Williams and C. B. Carter, Transmission Electron Microscopy I, (Plenum, New York 1996) p. 158.

9. S.M. Shapiro, B.X. Yang, Y. Noda, L.E. Tanner, D. Schryvers, Phys. Rev. B 44, 9301 (1991).

10. K. R. A. Ziebeck and P. J. Webster, J. Phys. F 5, 1756 (1975). 